\title{
HMGB1 suppresses colon carcinoma cell apoptosis triggered by co-culture with dendritic cells via an ER stress-associated autophagy pathway
}

\author{
YINGCHENG $\mathrm{ZHENG}^{1}$ and GUOGUO $\mathrm{ZHU}^{2}$ \\ Departments of ${ }^{1}$ Infection Control, and ${ }^{2}$ Emergency and Critical Care Medicine, \\ Wuhan General Hospital of Guangzhou Command, Wuhan, Hubei 430070, P.R. China
}

Received August 13, 2016; Accepted May 12, 2017

DOI: $10.3892 / \mathrm{mmr} .2017 .8202$

\begin{abstract}
High mobility group box protein 1 (HMGB1) is a versatile molecule that affects the immune system in various ways; however, its role in cancer immunity has not yet been completely elucidated. In the current study, bone marrow-derived dendritic cells from BALB/c mice and undifferentiated murine colon carcinoma CT26.WT cells were used as a cellular model to study the primary role of HMGB1 in colon cancer immunity. Annexin V and acridine orange/ethidium bromide staining was used to assess cellular apoptosis, Cell Counting kit 8 and lactate dehydrogenase assays were performed to evaluate cell viability and a monodansylcadaverine assay was used to detect autophagy. Western blot analysis was performed to detect the expression levels of proteins of interest. Endoplasmic reticulum (ER) stress and c-Jun N-terminal kinase phosphorylation were also investigated in CT26.WT cells exposed to dendritic cells. The present results demonstrated that the CT26.WT cells underwent apoptotic cell death following co-culturing with dendritic cells. However, pretreatment with HMGB1 resulted in a significant increase in viability of the CT26.WT cells exposed to dendritic cells. Furthermore, HMGB1 promoted ER stress-induced autophagy through the activation of JNK, which inhibited the apoptosis triggered by the dendritic cells,
\end{abstract}

Correspondence to: Dr Guoguo Zhu, Department of Emergency and Critical Care Medicine, Wuhan General Hospital of Guangzhou Command, 627 Luoyu Road, Wuhan, Hubei 430070, P.R. China E-mail: guoguoicetea@hotmail.com

Abbreviations: HMGB1, high mobility group box 1; ER, endoplasmic reticulum; JNK, c-Jun N-terminal kinases; DC, dendritic cell; APC, antigen presenting cell; TAA, tumor-associated antigen; LDH, lactate dehydrogenase; GM-CSF, granulocyte macrophage-colony stimulating factor; FBS, fetal bovine serum; LC3, microtubule-associated protein 1 light chain 3; GRP78, glucose regulated protein 78; PI3K, phosphoinositide 3-kinase

Key words: HMGB1, dendritic cell, ER stress, autophagy, JNK suggesting that HMGB1 has a role in immune evasion by colon cancer cells.

\section{Introduction}

Dendritic cells (DCs) are an important distinct subset of antigen-presenting cells (APCs) that have key roles in the uptake, progression and presentation of foreign and self-antigens, including tumor-associated antigens (TAAs) (1,2). Cancer-associated DCs present antigens derived from dying cancer cells to antigen-specific $\mathrm{CD}^{+}$cytotoxic $\mathrm{T}$ cells to induce cell-mediated immune responses against cancer (1). In turn, these responses directly induce cancer cell apoptosis via tumor necrosis factor-related apoptosis-inducing ligand, Fas and tumor necrosis factor- $\alpha$ receptors (3). Apoptosis triggered by APCs enhances TAA acquisition because apoptotic cell death leads to the release of TAAs from cancer cells. In addition, a group of host proteins, termed alarmins, function to 'warn' the innate and adaptive immune systems early and to interact with receptors that activate APCs, such as DCs (4).

High mobility group box protein 1 (HMGB1) is one such innate immune alarmin protein (5). It is a highly conserved non-histone chromatin-associated protein that functions as an architectural chromatin-binding protein and enables transcriptional protein assemblies to gain access to specific DNA targets (6). In addition to the role of HMGB1 in the nucleus as a chromatin stabilizing factor, it also functions as an extracellular signaling molecule. It is expressed in the nuclei of eukaryotic cells and is passively released by necrotic, apoptotic or autophagic cells (6). HMGB1 has roles in numerous biological processes, including inflammation, cell migration, cell differentiation and cancer metastasis $(7,8)$. It has been previously reported that HMGB1 is over-expressed in a variety of cancer cell types, and that it has central roles in carcinogenesis and cancer progression, invasion and metastasis by sustaining autophagy and limiting apoptosis (9). Furthermore, in the disordered microenvironment of cancer, HMGB1 recognition has a paradoxical effect on cancer immunity, promoting angiogenesis while inducing a protective anti-neoplastic $\mathrm{T}$ cell response (10).

Autophagy occurs at a low basal level in almost all cells in response to disordered microenvironmental stimuli, 
such as endoplasmic reticulum (ER) stress, mitochondrial toxins, hypoxia, abnormal cell growth, and nutrient deprivation (11). As part of the normal catabolic process required for homeostasis (12), autophagy has been implicated in many physiological and pathophysiological conditions (13). In response to stress, the upregulation of autophagy is a vital mechanism by which cells remove damaged proteins and organelles $(11,12)$. Autophagy has been shown to be important in conferring resistance to chemotherapy, radiation therapy and immunotherapy in cancer (14). The paradoxical dual effects of autophagy on carcinogenesis and cancer progression await further verification. As a response to disordered microenvironmental stimuli, autophagy can be activated by ER stress (11). Autophagy is activated sequentially following ER stress and saves cells undergoing ER stress (15). The mechanism by which ER stress activates autophagy is very complex. c-Jun N-terminal kinase (JNK) is a crucial mediator of the ER stress-induced autophagy pathway $(16,17)$. Multiple studies have reported that HMGB1 promotes autophagy by interfering with the binding of B-cell lymphoma $2(\mathrm{Bcl} 2)$ to Beclin $1(18,19)$; however, the function of HMGB1 release from cancer cells in response to immune cell-mediated stress remains unknown.

In the present study, it was demonstrated that HMGB1 is overexpressed in murine cancer cells and that it promotes the resistance of CT26.WT murine colon cancer cells to apoptosis triggered by murine bone marrow-derived DCs by promoting ER stress-induced autophagy. Furthermore, the role of exogenous HMGB1 in the regulation of autophagy depends on the activation of JNK, which links ER stress with autophagy. However, the suppression of HMGB1 expression or the depletion of HMGB1 by an HMGB1-neutralizing antibody in the culture medium increases the cytotoxicity of DCs to CT26.WT cells; this effect indicates that HMGB1 is a potential factor involved in cancer immune evasion.

\section{Materials and methods}

Mice and cell lines. Female BALB/c mice, 5-6 weeks old, were purchased from the Jackson Laboratory (Bar Harbor, ME, USA) and were bred in pathogen-free facilities in the Animal Biosafety Level-3 Lab of Wuhan University (Wuhan, China). All live animal experiments were approved by the Wuhan University Ethics Committee (Wuhan, China). The CT26.WT (murine colon cancer cells) and L929 cell (mouse immortal fibroblast cells) lines were purchased from the American Type Culture Collection (Manassas, VA, USA) and were cultures in RPMI-1640 medium (Invitrogen, Thermo Fisher Scientific, Inc., Waltham, MA, USA) supplemented with $100 \mathrm{U} / \mathrm{ml}$ penicillin (Sigma Aldrich; Merck KGaA, Darmstadt, Germany), $100 \mu \mathrm{g} / \mathrm{ml}$ streptomycin (Sigma Aldrich; Merck KGaA) and $10 \%$ fetal bovine serum (FBS; Gibco; Thermo Fisher Scientific, Inc.) in petri dishes or Transwell dishes (membrane pore size, $0.4 \mu \mathrm{m}$; Corning Incorporated, Corning, NY, USA). The cells were maintained at $37^{\circ} \mathrm{C}$ in a humidified atmosphere containing $5 \% \mathrm{CO}_{2}$ and were passaged every 2 days; the cell culture medium was changed once between the passages. The viability of the cells was determined using a trypan blue exclusion assay. For the cell viability and cytotoxicity assays, cells in the exponential growth phase were trypsinized and resuspended in fresh culture medium at a density of $5 \times 10^{3}$ cells/well in a 96 -well plate for $24 \mathrm{~h}$ at $37^{\circ} \mathrm{C}$. Following incubation, the medium was replaced with a suspension of DCs $\left(5 \times 10^{4}\right.$ cells/well), which were derived from murine bone marrow cells. For other experiments, the cells were trypsinized and resuspended in fresh culture medium at a density of $10^{5}$ cells/well in the sub-layer of the Transwell or petri dishes for $24 \mathrm{~h}$ at $37^{\circ} \mathrm{C}$. Following incubation, the medium was changed, and the DC suspension was added to the chamber.

Generation of bone marrow-derived DCs. Bone marrow derived DCs were prepared according to the protocol described by Lutz et al (20), with minor modifications. Briefly, the bone marrow cells of BALB/c mice were isolated from the femurs of adult mice and were cultured in RPMI-1640 medium supplemented with 10\% FBS (Gibco; Thermo Fisher Scientific, Inc.), $100 \mathrm{U} / \mathrm{ml}$ penicillin, $100 \mu \mathrm{g} / \mathrm{ml}$ streptomycin, $5 \mu \mathrm{M} \beta$-mercaptoethanol, $2 \mathrm{mM}$ L-glutamine (Hyclone; GE Healthcare Life Sciences, Logan, UT, USA), 20 ng/ml recombinant mouse granulocyte macrophage-colony stimulating factor (GM-CSF; R\&D Systems, Inc., Minneapolis, MN, USA) and $10 \mathrm{ng} / \mathrm{ml}$ IL-4 (Cell Signaling Technology, Inc., Danvers, MA, USA). On the third day of culture, all detached cells and loosely adherent cells were removed by gentle swirling, and fresh medium was added to the cells. On the 8th day, the non-adherent cells were collected for experiments, and the purity of the bone marrow-derived DCs was determined using flow cytometric analysis ( $>85 \% \mathrm{CD} 1 \mathrm{c}^{+}$cells). Cell viability was $>98 \%$, as determined by trypan blue exclusion assay.

Annexin $V$ assays. Annexin V assays were used to detect the CT26.WT cells that had externalized phosphatidylserine on the outer layer of the cell membrane, which is a marker of early apoptosis. The CT25.WT cells were exposed to DCs at a ratio of 1:10 for $4 \mathrm{~h}$. After removal of the DCs, the CT26.WT cells $\left(2 \times 10^{6}\right.$ cells $\left./ \mathrm{ml}\right)$ were stained with Annexin V conjugated to fluorescein isothiocyanate (FITC) and propidium iodide (PI) using the FITC Annexin V/Dead Cell Apoptosis kit (eBioscience; Thermo Fisher Scientific, Inc.) according to the manufacturer's protocol. Briefly, cells were suspended in $500 \mu \mathrm{l}$ Annexin $\mathrm{V}$ binding buffer and incubated with $5 \mu \mathrm{l}$ Annexin V and $5 \mu \mathrm{l}$ PI for $5 \mathrm{~min}$ at room temperature in the dark. Percentages of cells undergoing apoptosis were determined by dual-color flow cytometric analysis. Immediately after staining, the cells were analyzed on a flow cytometer using $488 \mathrm{~nm}$ as the excitation wavelength, a 525-nm band pass filter for FITC and a 620-nm filter for PI detection. At least 20,000 cells were acquired using a Beckman Coulter flow cytometer and data were analyzed using Beckman Summit software version 6.1.0 (Beckman Coulter, Inc., Brea, CA, USA).

Western blot analysis. The treated CT26.WT and L929 cells were harvested and lysed in radioimmunoprecipitation assay lysis buffer $(150 \mathrm{mM} \mathrm{NaCl}, 1 \% \mathrm{SDS}, 10 \mathrm{mg} / \mathrm{ml}$ leupeptin, $1 \mathrm{mM}$ aprotinin, $50 \mathrm{mM}$ Tris- $\mathrm{HCl}, \mathrm{pH} 7.4$ ) containing the protease inhibitor phenylmethylsulfonyl fluoride for $5 \mathrm{~min}$ on ice, prior to scraping and brief sonication. The cell lysates were cleared by a 15 -min centrifugation at $12,000 \mathrm{x} \mathrm{g}$ at $4^{\circ} \mathrm{C}$; the protein concentration was determined using a bicinchoninic 
acid protein assay kit (Pierce; Thermo Fisher Scientific, Inc.) and the samples were then boiled for $10 \mathrm{~min}$. Equal amounts of extracted protein samples $(10 \mu \mathrm{g})$ were loaded onto $10-12 \%$ gradient SDS-PAGE gels and run at $110 \mathrm{~V}$ at room temperature in SDS-PAGE running buffer (Sigma Aldrich; Merck KGaA). Following electrophoresis, the proteins were transferred to nitrocellulose membranes by electroblotting at $30 \mathrm{~V}$ in Tris/glycine buffer with $20 \%$ methanol for $1.5 \mathrm{~h}$ at $4^{\circ} \mathrm{C}$. The blots were blocked with $5 \%$ bovine serum albumin (cat. no. ST023; Beyotime Institute of Biotechnology, Haimen, China) in PBS washing buffer with $0.1 \%$ Tween-20 (PBST) for $3 \mathrm{~h}$ at room temperature. After rinsing with PBST (three times for $5 \mathrm{~min}$ ), the membranes were incubated with 1:1,000 dilutions of the following primary antibodies at $4^{\circ} \mathrm{C}$ overnight: HMGB1 (cat. no. 2639-1; Epitomics; Abcam, Cambridge, UK), microtubule-associated protein 1 light chain 3 (LC3; cat. no. 4108; Cell Signaling Technology, Inc.), sequestosome 1 (P62; cat. no. 3340-1; Epitomics; Abcam), glucose regulated protein 78 (GRP78; cat. no. 3158-1; Epitomics; Abcam), JNK (cat. no. 3496-1; Epitomics; Abcam), phospho (p)-JNK (cat. no. 3893-1; Epitomics; Abcam), p-Bcl2 (cat. no. 8588-1; Epitomics; Abcam), Bcl2 (cat. no. ab32124; Abcam), Beclin1 (cat. no. 2026-1; Epitomics; Abcam) or $\beta$-actin (cat. no. ab8226; Abcam). The membranes were then incubated with 1:1,000 dilutions of horseradish peroxidase-conjugated secondary antibody (cat. no. ab191866; Abcam) for $1.5 \mathrm{~h}$ at room temperature. The bound antibodies were visualized using the ECL Supersignal West Pico Trial Kit (Pierce; Thermo Fisher Scientific, Inc.). Densitometry analysis was performed using the ImageJ software version 1.429 (National Institutes of Health, Bethesda, MA, USA). The signal density was normalized to the expression of b-actin, which was used as a loading control; at least three separate experiments were analyzed.

Cytotoxicity assays. Lactate dehydrogenase (LDH) release was measured using the CytoTox 96 Non-Radioactive Cytotoxicity Assay kit (Promega Corporation, Madison, WI, USA) according to the manufacturer's instructions. HMGB1 pretreated or untreated CT26.WT cells were seeded at a density of 5,000 cells/well in 96-well plates in $100 \mu$ l complete RPMI-1640 medium and were incubated overnight. DCs were added to the CT26.WT cells at a ratio 1:10. The DCs alone and the supernatant of the co-cultures mentioned above were used as controls. After $4 \mathrm{~h}$, the supernatants were collected, and the resulting LDH release of the CT26.WT cells was determined.

Cell viability assay. The CT26.WT cells were seeded in 96-well plates at a density of 5,000 cells/well under each condition. The murine bone marrow-derived DCs were then added to the wells at a ratio $1: 10$. The plates were incubated at $37^{\circ} \mathrm{C}$ with $5 \% \mathrm{CO}_{2}$ for $24 \mathrm{~h}$ and were then subjected a Cell Counting Kit-8 (CCK-8) assay using the CCK-8 assay kit (Beyotime Institute of Biotechnology) according to the manufacturer's instructions.

Acridine orange (AO)/ethidium bromide (EB) cell apoptosis assay. A total of $25 \mu 1$ of CT26.WT cell suspension $\left(0.5-2.0 \times 10^{6}\right.$ cells $\left./ \mathrm{ml}\right)$ were incubated with $1 \mu \mathrm{l} \mathrm{AO} / \mathrm{EB}$ solution at $4^{\circ} \mathrm{C}$ for $1 \mathrm{~min}$ in darkness. Samples were evaluated immediately by placing $10 \mu \mathrm{l}$ cell suspension onto a microscope slide, covering with a glass coverslip, and examined under a fluorescence microscope (Olympus CX31; Olympus Corporation, Tokyo, Japan) using a fluorescein filter at $510 \mathrm{~nm}$ and an x40 objective. At least 300 cells were observed.

Monodansylcadaverine (MDC) staining. To evaluate autophagy, MDC powder was purchased from Sigma Aldrich (Merck KGaA) and dissolved in dimethyl sulfoxide at $0.1 \mathrm{~mol} / 1$ of the stock concentration; the working concentration was $50 \mu \mathrm{m} / 1$. The CT26.WT cells $\left(1 \times 10^{6}\right.$ cells $\left./ \mathrm{ml}\right)$ were incubated with the MDC dye for $45 \mathrm{~min}$ in the dark at $4^{\circ} \mathrm{C}$ and then washed with PBS thrice. Subsequently, they were visualized under a fluorescence microscope (Olympus Corporation). The autophagosomes emitted blue fluorescence and appeared in a punctate pattern in CT26.WT cells.

Pharmacological and antibody interventions. The following inhibitors were used in the present study: ER stress inhibitor 4-phenylbutyrate (4-PBA; 10 mM; EMD Millipore, Billerica, MA, USA) and JNK phosphorylation inhibitor SP600125 (10 $\mu \mathrm{M}$; Selleck Chemicals, Houston, TX, USA). The drugs were dissolved in 10\% FBS in RPMI-1640 medium and were added to the CT26.WT cell cultures 30 min prior to initiating the DC exposure. HMGB1 antibody (cat. no. 2639-1; Epitomics; Abcam) 1:50 (antibody/culture v/v) was applied to neutralize HMGB1 in cell cultures, and rabbit IgG monoclonal antibody (cat. no. ab199376; Abcam; 1:50) was used as its isotype control.

RNA transfection. Small interfering (si)RNA duplexes specifically targeting HMGB1 and control siRNAs were obtained from Abgent Biotech Co., Ltd. (Suzhou, China) and $100 \mathrm{nM}$ siRNA was transfected into $5 \times 10^{5}$ CT26.WT cells using Lipofectamine 2000 (Invitrogen; Thermo Fisher Scientific, Inc.) for $24 \mathrm{~h}$ in $37^{\circ} \mathrm{C} \mathrm{CO}_{2}$ incubator, according to the manufacturer's protocol. The following RNA oligonucleotides were used in the present study: Control siRNA, 5'-GUUAUCGCA ACGUGUCACGUA-3'; HMGB1 siRNA, 5'-GCAGCCCUA UGAGAAGAAATT-3'.

Statistical analysis. The results are presented as the mean \pm standard deviation. Experiments were repeated at least 3 times. Comparisons between two groups were analyzed using Student's t-test, whereas comparisons among multiple groups were analyzed using a one-way analysis of variance followed by a post hoc Tukey test. $\mathrm{P}<0.05$ was considered to indicate a statistically significant difference.

\section{Results}

HMGB1 is associated with the viability of CT26.WT cells exposed to DCs. To determine the role of HMGB1 in the colon cancer cell response to DC exposure, HMGB1 expression in the murine colon cancer cell line CT26.WT. As demonstrated in Fig.1A,HMGB1 expression was markedly higher in CT26.WT cells compared with the L929 immortalized murine fibroblast cell line, as determined by immunoblot analysis, indicating that HMGB1 expression may be upregulated in murine colon cancer cells (Fig. 1A). Subsequently, a specific siRNA targeting HMGB1 was used to knock down its expression in CT26.WT 
A

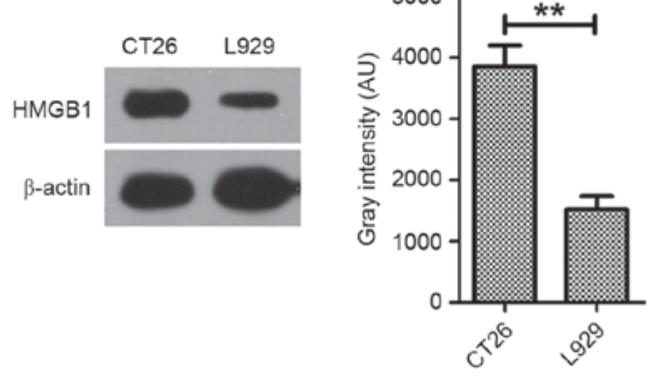

C

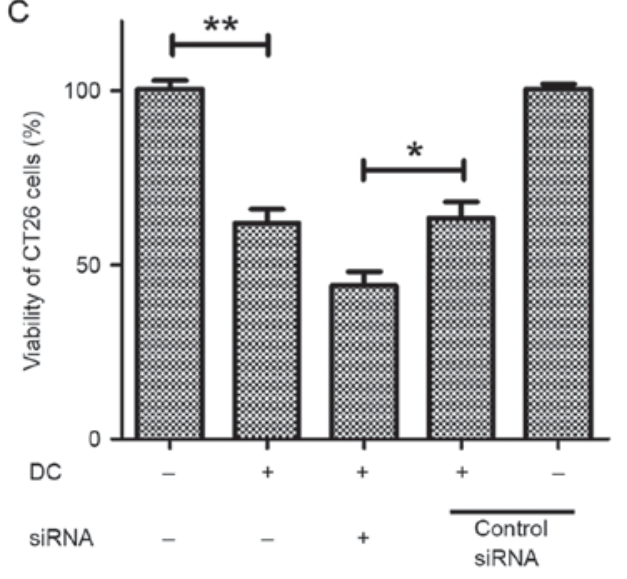

$\mathrm{E}$

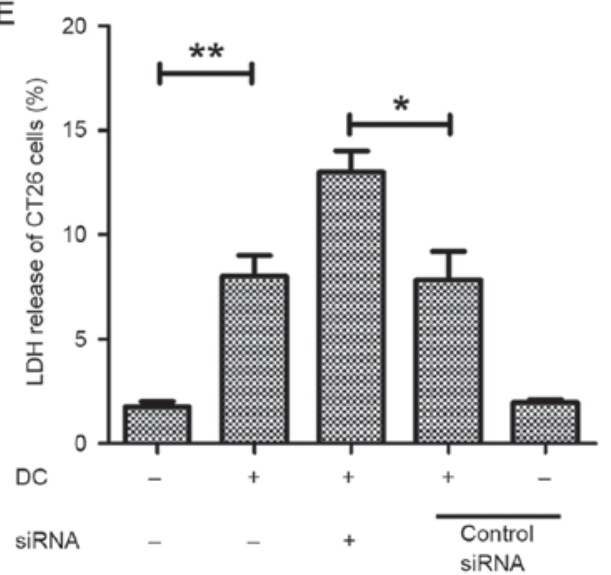

B
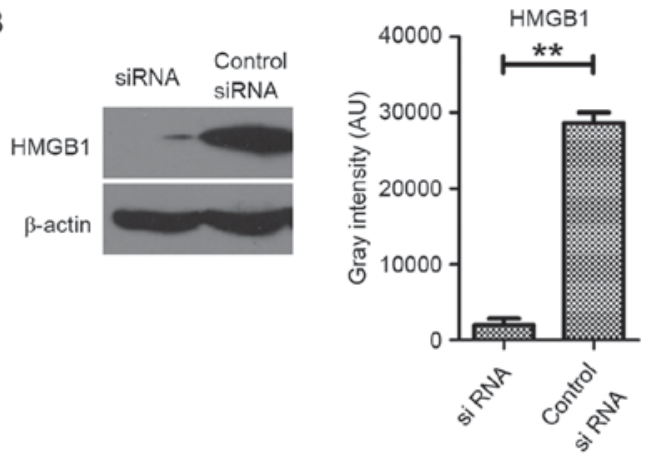

D

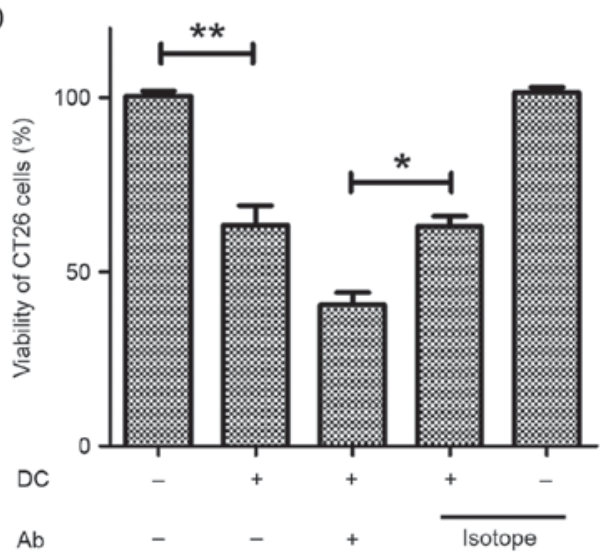

$\mathrm{F}$

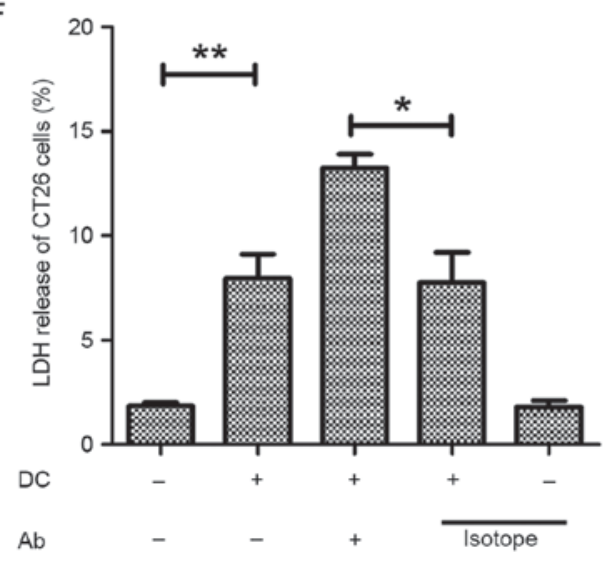

Figure 1. HMGB1 is associated with the viability of CT26.WT cells exposed to murine bone marrow-derived DCs. (A) HMGB1 expression in the murine colon cancer cell line CT26.WT and L929 cells was measured by western blot analysis (n=4). (B) HMGB1 expression was efficiently inhibited by a specific siRNA targeting HMGB1 in CT26.WT cells, as determined by western blot analysis $(n=4)$. The viability of HMGB1-deficient CT26.WT cells was determined by a CCK-8 assay in CT26.WT cells after exposure to DCs for $24 \mathrm{~h}$ at a ratio of 1:10; cells were also exposed to (C) siRNA targeting HMGB1 or (D) HMGB1-neutralizing antibody $(1: 50)(\mathrm{n}=8)$. LDH release was measured following treatment of CT26.WT cells with DCs at a ratio of 1:10 for $24 \mathrm{~h}$ and (E) an siRNA targeting HMGB1 or (F) HMGB1-neutralizing antibody $(\mathrm{n}=8)$. The data represent the mean \pm standard deviation. $\mathrm{P}<0.05,{ }^{* *} \mathrm{P}<0.01$. HMGB1, high mobility group box protein 1 ; AU, arbitrary units; siRNA, small interfering RNA; DC, dendritic cell; Ab, HMGB1-neutralizing antibody; LDH, lactate dehydrogenase.

cells (Fig. 1B). The viability of HMGB1-deficient CT26.WT cells exposed to DCs was significantly lower than that of control siRNA CT26.WT cells exposed to the DCs (Fig. 1C). Additionally, an HMGB1-neutralizing antibody (1:50) was used to reduce the concentration of HMGB1 in the supernatant, which resulted in significantly decreased viability of the CT26.WT cells exposed to the DCs compared with those exposed to DCs and an isotypeantibody control(Fig. 1D). Theseresults indicated that HMGB1 has an important role protecting the CT26.WT cells from the effects of DCs. Additionally, the cytotoxic effect of DCs on CT26.WT cells was significantly reduced following the siRNA-mediated knockdown of HMGB1 or its depletion using a neutralizing antibody, as measured by LDH release (Fig. 1E and F). These results indicate that HMGB1 is highly important for maintaining the viability of CT26.WT cells exposed to DCs.

HMGB1 increases the resistance of CT26.WT cells to apoptosis after exposure to DCs. Further experiments were conducted to determine whether HMGB1 is able to prevent apoptosis of CT26.WT cells exposed to DCs. Following pretreatment with $10 \mathrm{ng} / \mathrm{ml} \mathrm{HMGB1}$, the viability of DC-exposed CT26.WT cells was significantly increased compared with that of cells that were not pretreated, as measured by CCK-8 assay (Fig. 2A). 
A

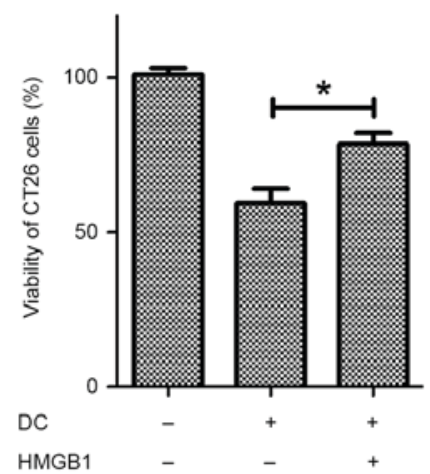

B
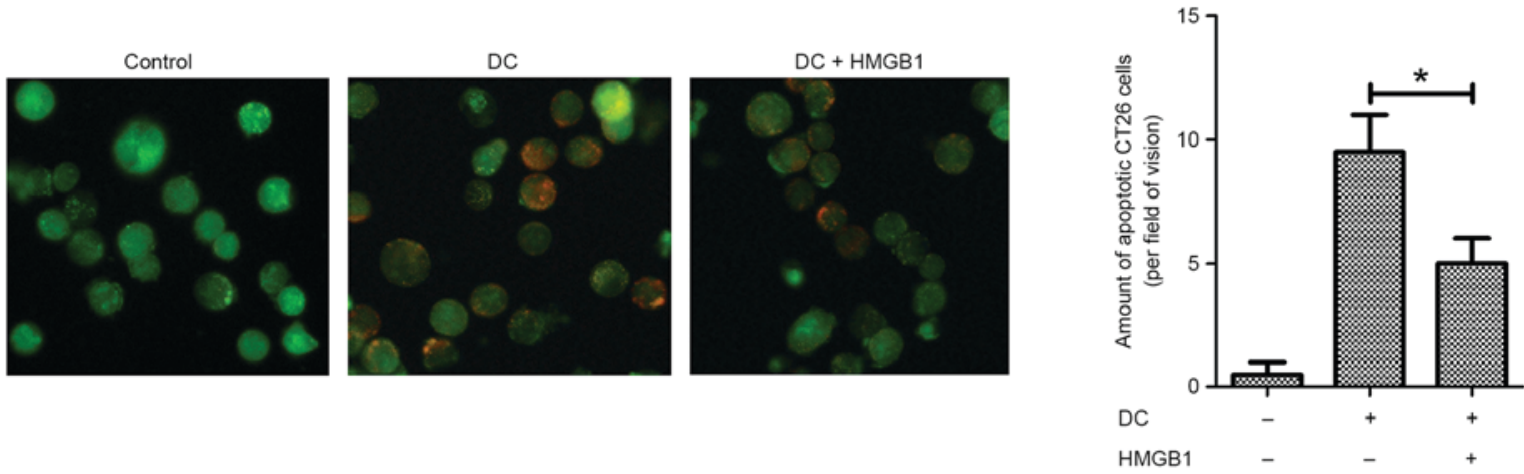

C
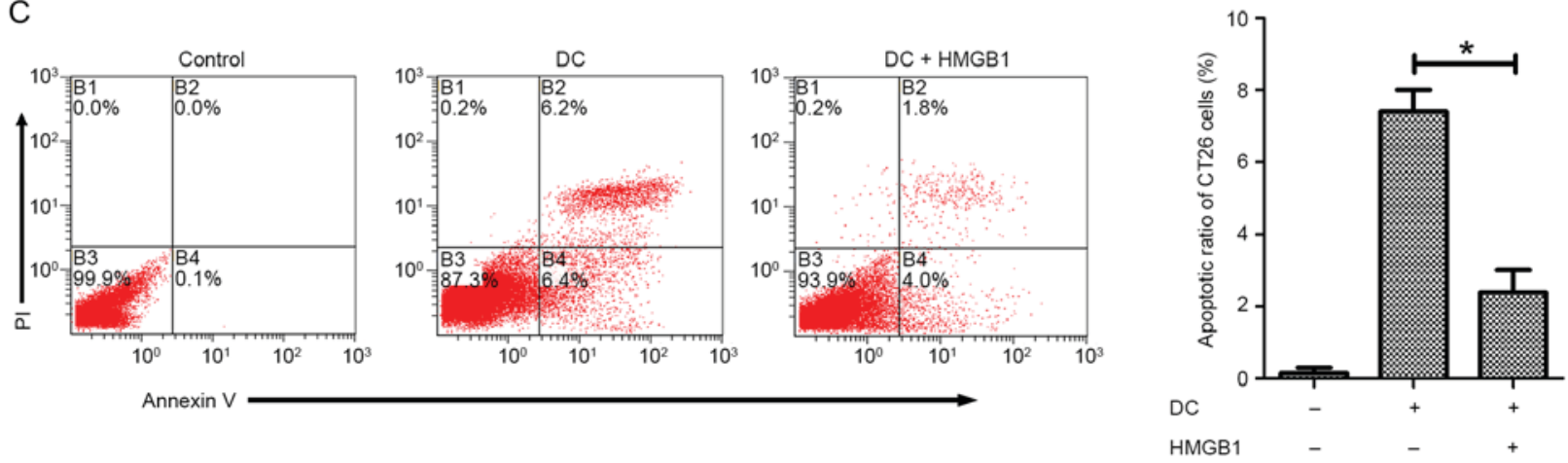

Figure 2. HMGB1 inhibits apoptotic cell death in CT26.WT cells exposed to DCs. (A) Following pretreatment with $10 \mathrm{ng} / \mathrm{ml}$ HMGB1 for 24 h, cell viability was measured in CT26.WT cells exposed to DCs at a ratio of 1:10 for $24 \mathrm{~h}$ and compared with that of non-pretreated cells. (B) CT26.WT cells pretreated with $10 \mathrm{ng} / \mathrm{ml} \mathrm{HMGB} 1$ for $24 \mathrm{~h}$ were exposed to DCs at a ratio of 1:10 for $24 \mathrm{~h}$ and were then stained with acridine orange/ethidium bromide; orange-stained CT26.WT cells were considered non-viable, apoptotic cells. Magnification, x400. (C) The proportion of apoptotic CT26.WT cells was determined by Annexin V assay following pretreatment with $10 \mathrm{ng} / \mathrm{ml}$ HMGB1 for $24 \mathrm{~h}$ and exposure to DCs at a ratio of 1:10 for $24 \mathrm{~h}$. The data represent the mean \pm standard deviation ( $\mathrm{n}=6)$. ${ }^{*} \mathrm{P}<0.05$. DC, dendritic cell; HMGB1, high mobility group box protein 1; PI, propidium iodide.

Furthermore, an acridine orange/ethidium bromide cell apoptosis assay revealed that pretreatment with HMGB1 significantly reduced the number of non-viable, apoptotic CT26.WT cells after exposure to DCs and postponed apoptosis (Fig. 2B). To confirm these findings, an Annexin V assay was performed, demonstrating similar results (Fig. 2C). These findings suggest that HMGB1 enhances the viability of CT26.WT cells by preventing DC-induced apoptosis.

HMGB1 enhances the viability of CT26.WT cells exposed to DCs through the upregulation of autophagy. It has been suggested that two processes simultaneously occur during programmed cell death: Autophagy and apoptosis. Increasing evidence suggests that autophagy is an important mechanism by which cells resist stress. The aforementioned results suggest that HMGB1 promotes the resistance of CT26.WT cells to stress induced by DCs, leading to speculation that HMGB1 may influence autophagy in CT26.WT cells exposed to DCs. To verify this hypothesis, the conversion of LC3 and the degradation of P62 were measured by immunoblot analysis. Following pretreatment with $10 \mathrm{ng} / \mathrm{ml}$ HMGB1, autophagic flux was significantly increased in DC-exposed CT26.WT cells (Fig. 3A). This increased autophagic flux was confirmed by MDC staining, which specifically labels autophagic vacuoles. The mean fluorescence intensity of the DC-exposed CT26. WT cells was obviously increased following pretreatment with HMGB1 compared with cells exposed to DCs only, as shown by fluorescence microscopy (Fig. 3B). Mechanistically, a significant increase in the expression of Beclin 1, a key molecule required for the initiation of autophagosomes, was detected in the DC-exposed CT26.WT cells following pretreatment with HMGB1 compared with those that did not receive pretreatment. Increased phosphorylation of $\mathrm{Bcl} 2$ was also observed, suggesting the disassociation of Beclin 1 
A
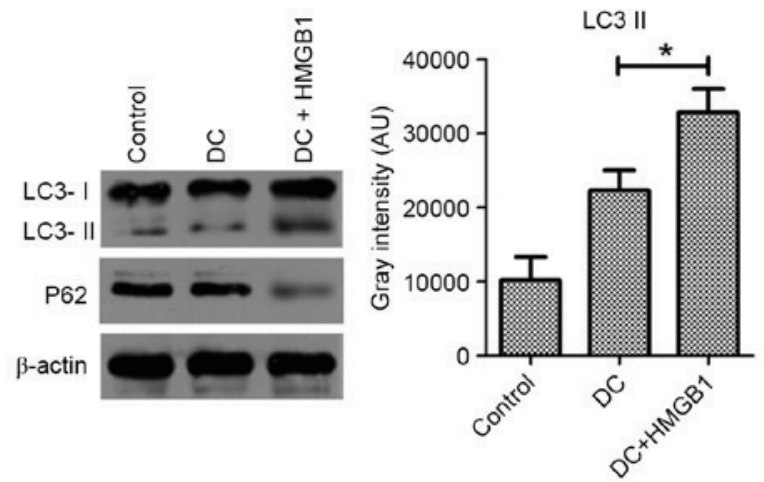

P62

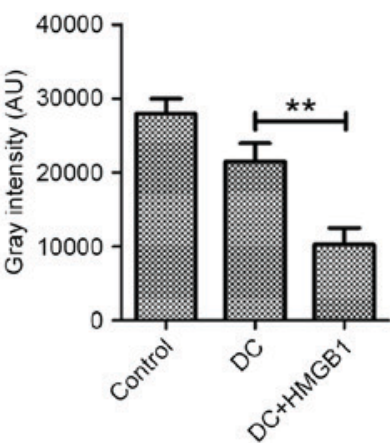

B
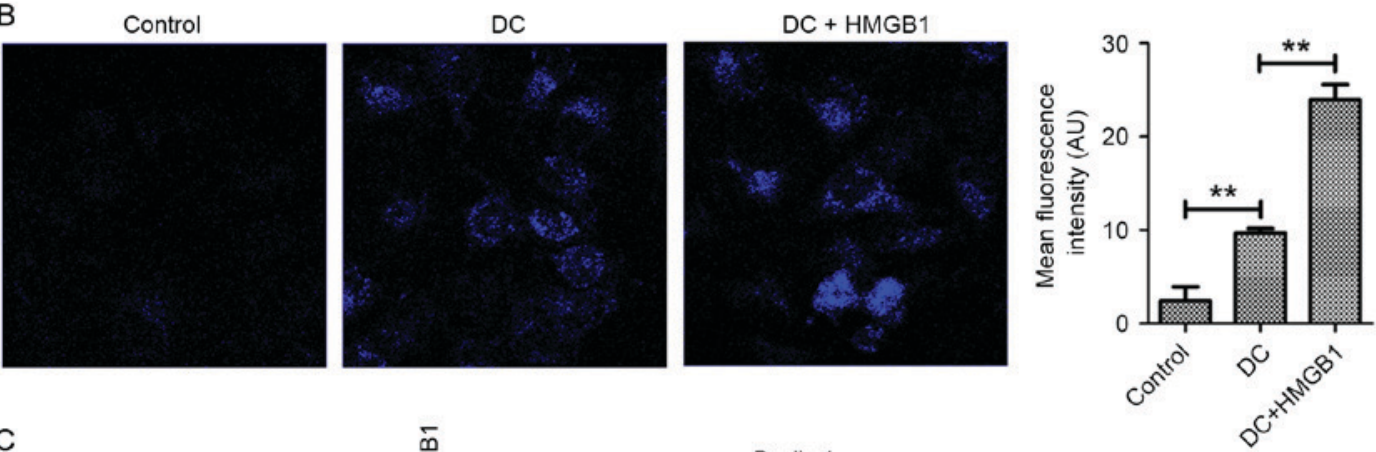

C
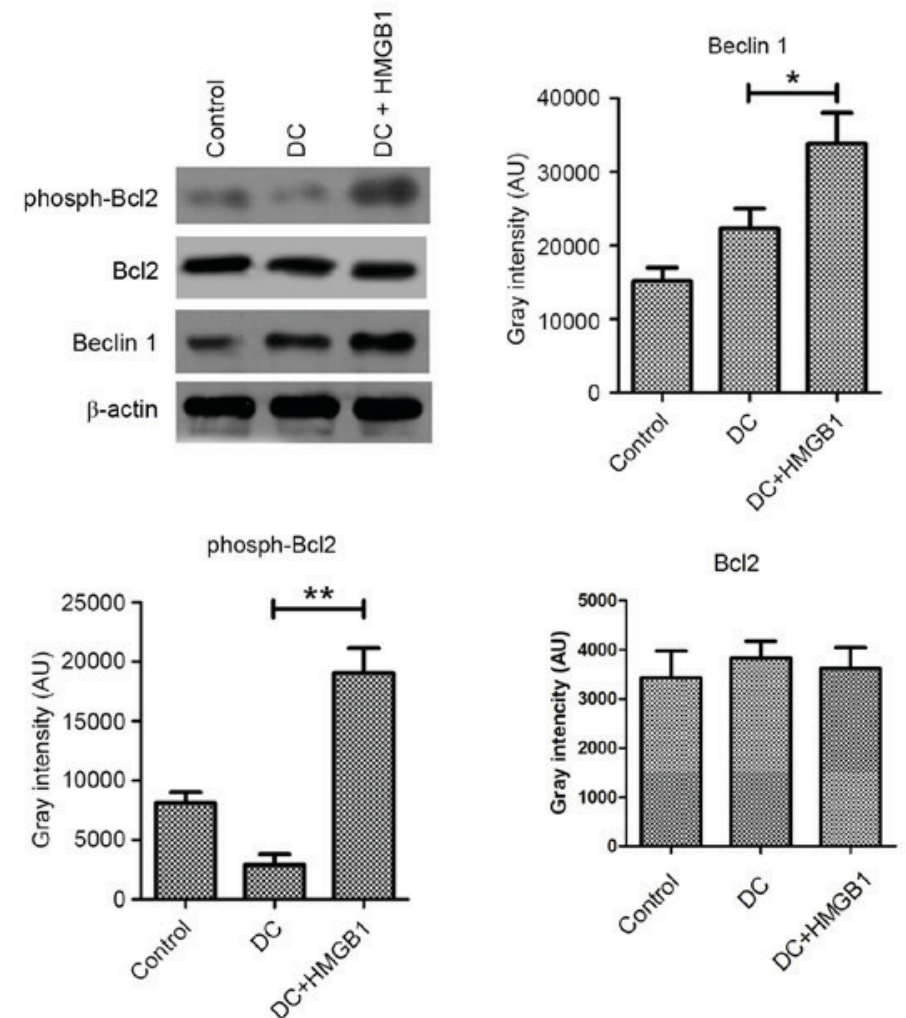

Figure 3. HMGB1 upregulates autophagy in CT26.WT cells exposed to DCs. (A), Pretreatment with $10 \mathrm{ng} / \mathrm{ml}$ HMGB1 increased the conversion of LC3 and the degradation of P62 in CT26.WT cells exposed to DCs at a ratio of 1:10 for $24 \mathrm{~h}$ compared with non-pretreated cells, as determined by immunoblot analysis. (B) Monodansylcadaverine was used to label autophagic vesicles, and the fluorescence intensity of the CT26.WT cells exposed to DCs at a ratio of 1:10 for $24 \mathrm{~h}$ was determined by fluorescence microscopy. Magnification, x400. (C) The levels of Beclin 1 expression and phosphorylated Bcl2 were determined by immunoblot analysis in $10 \mathrm{ng} / \mathrm{ml}$ HMGB1-pretreated CT26.WT cells exposed to DCs at a ratio of 1:10 for $24 \mathrm{~h}$. The data represent the mean \pm standard deviation $(\mathrm{n}=4) .{ }^{*} \mathrm{P}<0.05,{ }^{* *} \mathrm{P}<0.01$. LC3, microtubule-associated protein 1 light chain 3; P62, sequestosome 1; DC, dendritic cell; HMGB1, high mobility group box protein 1; AU, arbitrary units; Bcl2, B-cell lymphoma 2.

and Bcl2 complexes. All of these data suggest that HMGB1 induces autophagy in CT26.WT cells exposed to DCs.

ER stress is involved in HMGB1-induced autophagy in CT26. WT cells. Given that ER stress is an important disordered microenvironmental stimulus for autophagy, it was aimed to determine the role of ER stress in HMGB1-induced autophagy of CT26.WT cells exposed to DCs. The expression of GRP78, a hallmark of ER stress, was apparently increased in the HMGB1-treated, DC-exposed CT26.WT cells (Fig. 4A), 
A

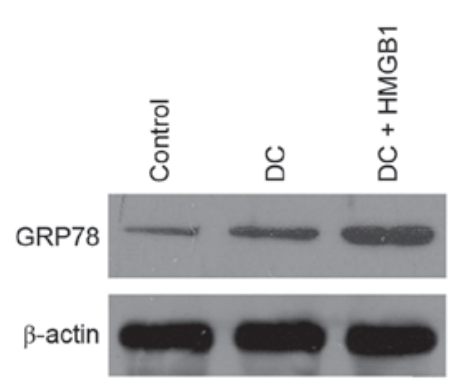

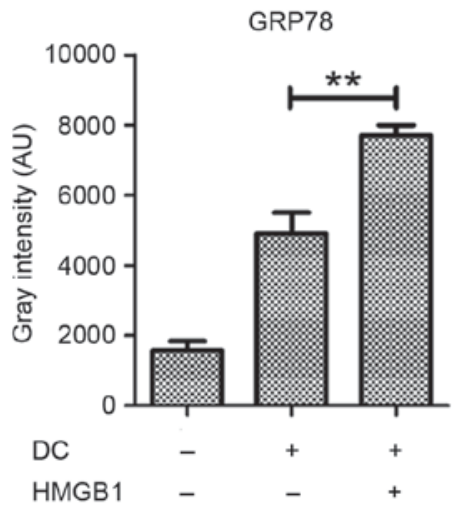

B
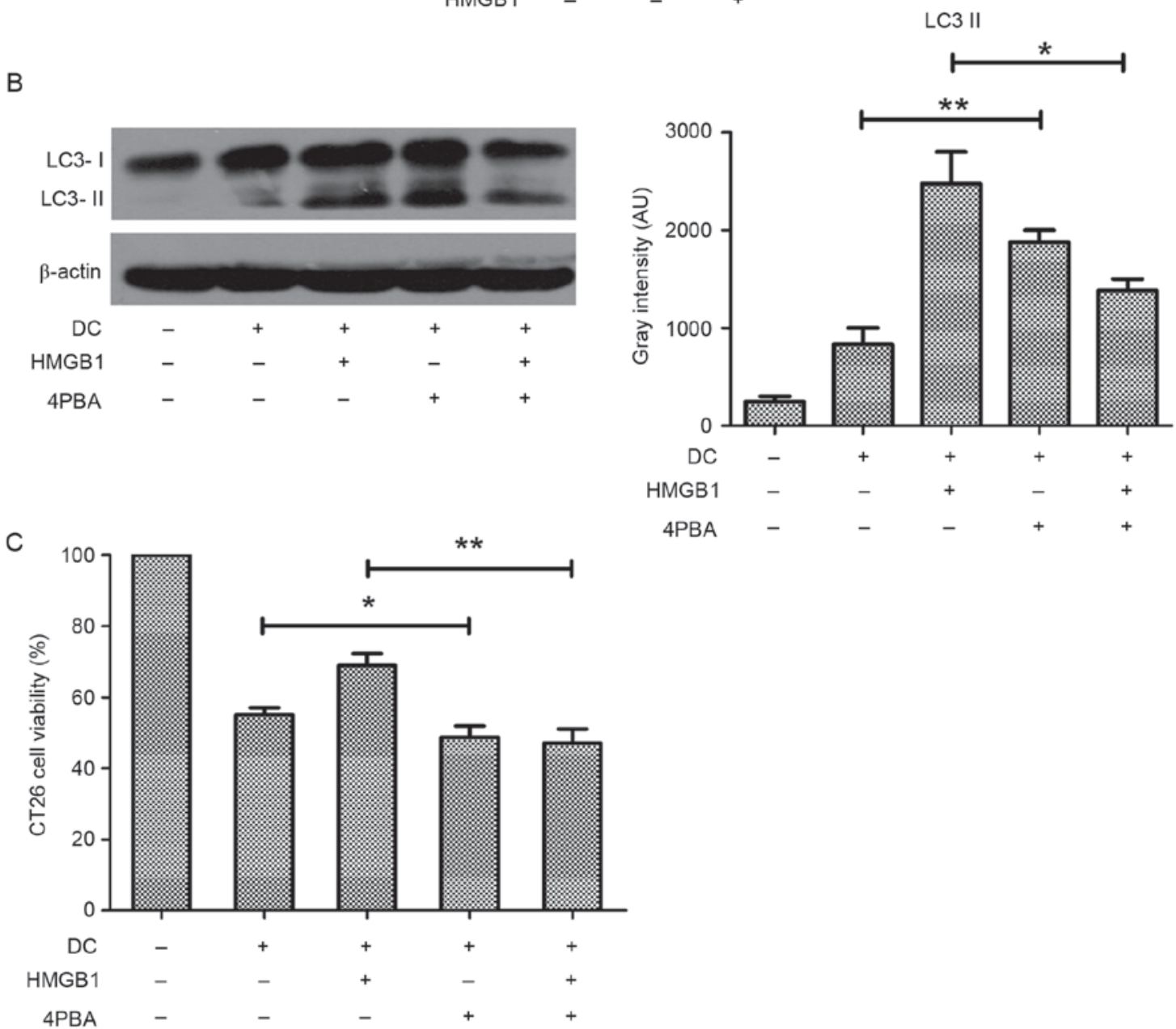

Figure 4. ER stress is associated with HMGB1-induced autophagy in CT26.WT cells exposed to DCs. (A) GRP78 expression was determined by immunoblot analysis of CT26.WT cells pretreated with $10 \mathrm{ng} / \mathrm{ml}$ HMGB1 and exposed to DCs at a ratio of 1:10 for $24 \mathrm{~h}(\mathrm{n}=4)$. (B) HMGB1-pretreated CT26.WT cells were treated with $10 \mathrm{mM}$ 4-PBA for $30 \mathrm{~min}$ and then exposed to DCs at a ratio of 1:10 for $24 \mathrm{~h}$; LC3 conversion was determined by immunoblot analysis (n=4). (C) Treatment with $10 \mathrm{mM}$ 4-PBA significantly decreased the viability of HMGB1-pretreated CT26.WT cells exposed to DCs at a ratio of 1:10 for 24 h, as determined by CCK- 8 assay $(n=8)$. The data represent the mean \pm standard deviation. "P $<0.05,{ }^{* *} \mathrm{P}<0.01$. DC, dendritic cell; HMGB1, high mobility group box protein 1; GRP78, glucose regulated protein 78; AU, arbitrary units; LC3, microtubule-associated protein 1 light chain 3; 4-PBA, 4-phenylbutyrate.

suggesting that HMGB1 promoted ER stress in these cells. Whereas HMGB1 pretreatment resulted in a marked increase in the conversion of LC3, treatment with $10 \mathrm{mM}$ 4-PBA to block ER stress reduced this conversion in the CT26.WT cells exposed to DCs (Fig. 4B), suggesting that ER stress was involved in HMGB1-induced autophagy in these cells. Consistently, CCK-8 assay demonstrated that treatment with 4-PBA resulted in a significant decrease in cell viability (Fig. 4C). These results suggest that ER stress mediates HMGB1-induced protective autophagy in CT26.WT cells exposed to DCs.
JNK phosphorylation links ER stress to HMGBl-induced autophagy. Activated JNK phosphorylates $\mathrm{Bcl} 2$ to facilitate disassociation of the Beclin 1-Bcl2 complex, which is essential for the initiation of autophagosomes. The phosphorylation of $\mathrm{Bcl} 2$ was increased in the HMGB1-pretreated CT26.WT cells, suggesting the involvement of JNK kinase activity in HMGB1-induced autophagy. To verify this, JNK phosphorylation in HMGB1-pretreated, DC-exposed CT26.WT cells was evaluated and observed that JNK phosphorylation was increased by DCs and further increased by 
A
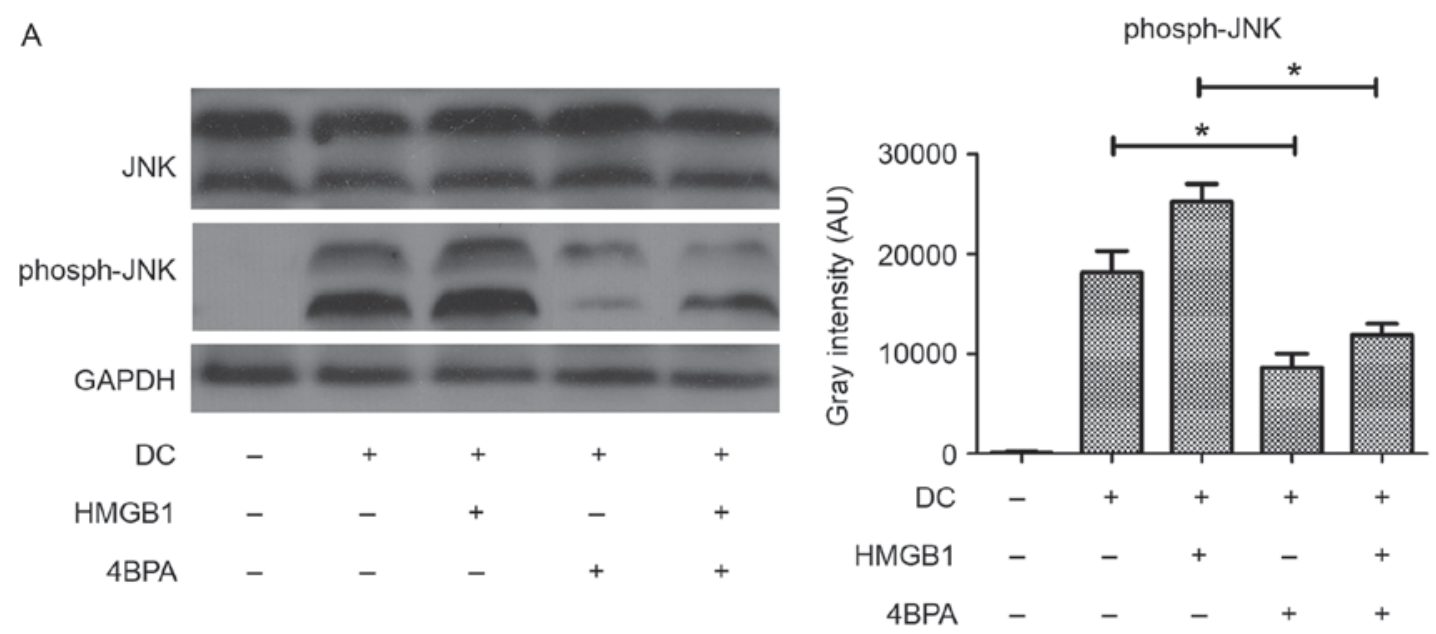

B
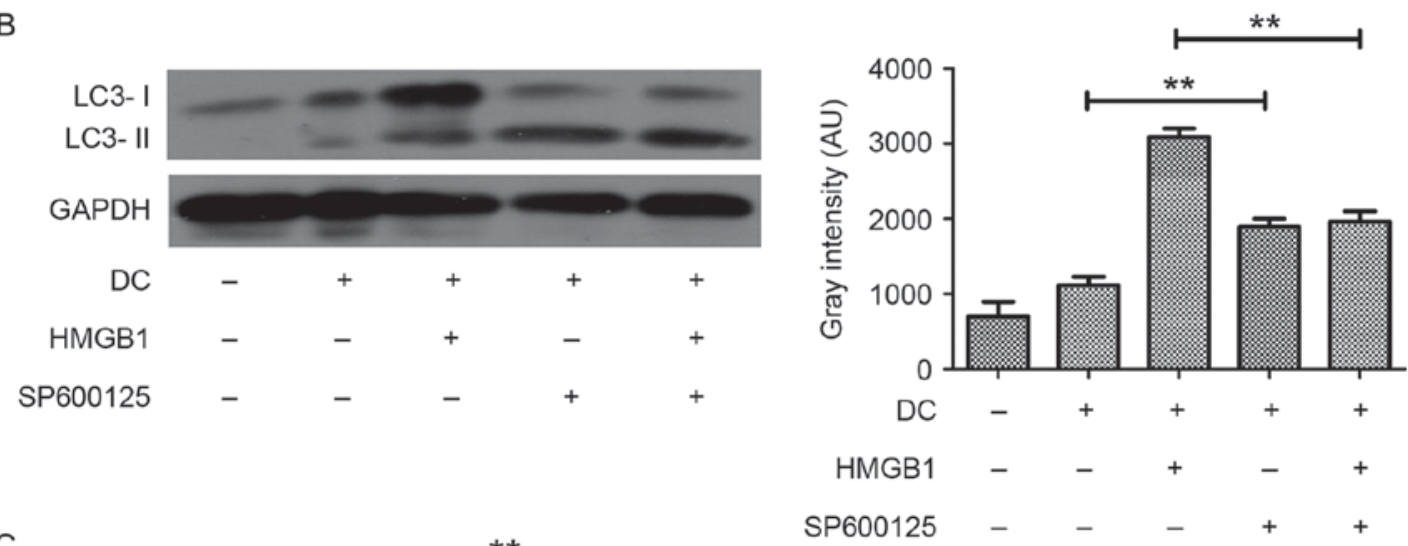

C

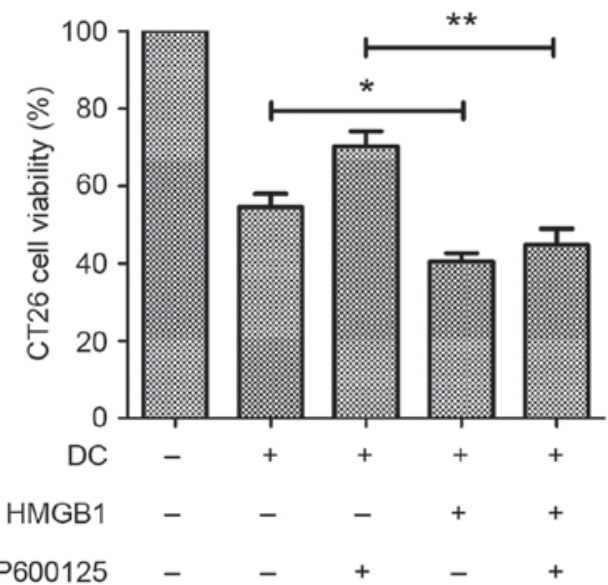

Figure 5. JNK phosphorylation is upregulated by HMGB1 and links ER stress to autophagy. (A) JNK phosphorylation was determined in CT26.WT cells exposed to DCs at a ratio of 1:10 for $24 \mathrm{~h}$. Cells were pretreated with $10 \mathrm{ng} / \mathrm{ml}$ HMGB1 and with $10 \mathrm{mM}$ 4-PBA for 30 min prior to DC exposure. JNK phosphorylation decreased in the HMGB1-pretreated CT26.WT cells exposed to DCs, as determined by immunoblot analysis (n=4). (B) Conversion of LC3-II was determined by immunoblot analysis in HMGB1-pretreated CT26.WT cells exposed to DCs at a ratio of 1:10 and $10 \mu \mathrm{M} \mathrm{JNK}$ inhibitor SP600125 (n=4). (C) Viability of the HMGB1-pretreated CT26.WT cells that were exposed to DCs at a ratio of 1:10 for $24 \mathrm{~h}$ and $10 \mu \mathrm{M}$ SP600125 was measured using a CCK-8 assay $(\mathrm{n}=8)$. Data represent the mean \pm standard deviation. ${ }^{*} \mathrm{P}<0.05,{ }^{* * *} \mathrm{P}<0.01$. JNK, c-Jun $\mathrm{N}$-terminal kinase; DC, dendritic cell; HMGB1, high mobility group box protein 1; 4-PBA, 4-phenylbutyrate; LC3, microtubule-associated protein 1 light chain 3.

HMGB1 pretreatment (Fig. 5A). To clarify the association between JNK activation, HMGB1-induced autophagy and ER stress, 4-PBA was used to inhibit stress, and the resulting effect on JNK phosphorylation was assessed. Treatment of DC-exposed CT26.WT cells with $10 \mathrm{mM}$ 4-PBA resulted in inhibition of JNK phosphorylation (Fig. 5A). In addition, the increased conversion of LC3 induced by HMGB1 was inhibited by treatment with the JNK inhibitor SP600125, suggesting that JNK activation may link ER stress with autophagy in the CT26.WT cells exposed to DCs (Fig. 5B). In addition, treatment of HMGB1-pretreated CT26.WT cells exposed to DCs with SP600125 also significantly decreased their viability (Fig. 5C). These results suggest that HMGB1-induced autophagy is activated by ER stress through JNK phosphorylation in CT26.WT cells exposed to DCs. 


\section{Discussion}

Immune evasion is an essential step in cancer progression. One principle of immune evasion by cancer cells is that these cells suppress the immune system, resulting in failure of the immune system to detect them through TAAs, thereby enhancing their survival. Following exposure of cancer cells to immunological stress, they acquire phenotypes that allow them to escape from immune surveillance (21). This process involves dysfunction of the antigen presenting and progressing abilities of APCs such as DCs. DCs, the most efficient APCs, are critical for cancer antigen processing and presentation and have the extraordinary ability to induce cancer-directed $\mathrm{T}$ cell responses; therefore, these cells have the potential to be used in biotherapy (22). However, there are always obstacles to overcome. Some cancer cell phenotypes inhibit the immune pathway, and one therapeutic strategy involves impeding the acquisition of TAAs by APCs such as DCs $(23,24)$. Apoptosis results in the release of cancer cell contents, including many TAAs. Therefore, if cancer cells elude DC-induced apoptosis, they may partially contribute to immune system surveillance and immune evasion. The results of this study have demonstrated that HMGB1 is overexpressed in the colon cancer cell line CT26.WT and that HMGB1 expression enables CT26.WT cells to avoid apoptotic cell death triggered by bone marrow-derived dendritic cells. Our results have also shown that HMGB1 treatment reduces the cytotoxicity of DCs to CT26.WT cells, indicating that HMGB1 is closely associated with cancer immune evasion.

HMGB1 is overexpressed in a variety of types of cancer cells, and it can help cancer cells to cope with stresses induced by chemotherapy (25) or immune cytokines, resulting in their survival; thus, HMGB1 has been demonstrated to be closely associated with chemotherapy resistance and cancer evasion. A previous study demonstrated that the cancer-associated expression of the receptor T-cell immunoglobulin and mucin-domain containing-3 in DCs results in inhibition of the anticancer efficacy of DNA vaccines and chemotherapy through its binding to the damage-associated molecular pattern molecule HMGB1 (26). HMGB1 produced by cancer cells has an inhibitory effect on DCs in mice and humans (27). Studies examining the mechanisms by which HMGB1 contributes to carcinogenesis have been largely focused on molecular biological and biochemical aspects and therapeutic strategies based on the clinical targeting of HMGB1. The results of this study revealed that HMGB1 is a damage-associated molecular pattern molecule that rescues CT26.WT cells from stress caused by DCs by promoting autophagy. siRNA-mediated depletion of HMGB1 or antibody-mediated neutralization in the co-culture supernatant resulted in decreased autophagy of CT26.WT cells exposed to DCs and increased the vulnerability of these cells to the DCs. Thus, we hypothesize that HMGB1 helps CT26.WT cells escape from apoptotic cell death by promoting the autophagic pathway. Beclin 1 has a central role in autophagy and is important for the localization of autophagic proteins to pre-autophagosomes, depending on its interaction with class 3 phosphoinositide 3 kinase (PI3K) (18). The current study also demonstrated that HMGB1 is a direct regulator of autophagy in CT26.WT cells and that it promotes LC3 conversion, P62 degradation, Beclin 1 upregulation and increased $\mathrm{Bcl} 2$ phosphorylation, which are key molecular events in the formation of the PI3KIII/Beclin 1 complex. Taken together, these results indicate that HMGB1-induced autophagy is dependent upon class 3 PI3K/Beclin 1.

ER stress is a critical disordered microenvironmental stimulus for autophagy. The results of the current study demonstrated that GRP78 expression was increased following pretreatment with HMGB1 in CT26.WT cells exposed to DCs and that it was suppressed by treatment with the ER stress inhibitor 4-PBA. HMGB1 treatment also increased the conversion of LC3 I to LC3 II and the degradation of P62 in the CT26.WT cells exposed to DCs, suggesting that ER stress is involved in HMGB1-induced autophagy. The pathways that link ER stress with autophagy are complex, and one important mechanism involves the phosphorylation of JNK, which is important for cell survival (27). As demonstrated in the present study, JNK phosphorylation was increased following treatment with HMGB1 in the CT26.WT cells exposed to DCs and that it was suppressed by 4-PBA treatment. In addition, treatment of these cells with the JNK inhibitor SP600125 inhibited the conversion of LC3 and the degradation of P62.

In conclusion, HMGB1 functions as an autophagy effector in CT26.WT cells exposed to DCs and promotes autophagy by increasing ER stress through JNK phosphorylation and the PI3K class 3/Beclin 1 complex to promote resistance to DC-induced apoptotic cell death. However, the specific region of HMGB1 that binds to Beclin 1 and how this binding affects downstream genes in CT26.WT cells are still largely unknown. Further studies are required to address these questions. The results of the study suggest that HMGB1 is released from CT26.WT cells during cancer immune evasion and that it functions as a positive regulator of autophagy that prevents DC-induced apoptosis. The findings also indicate that HMGB1 activates class $3 \mathrm{PI} 3 \mathrm{~K} /$ Beclin 1 , thereby regulating autophagosome formation and the fusion of autophagosomes with lysosomes. In light of promising autophagy inhibitors that increase the susceptibility of cancer cells to conventional therapies, the findings also suggest that HMGB1 is a crucial regulator of autophagy in CT26.WT cells exposed to DCs. These findings may facilitate the development of novel treatment strategies for colon cancer.

\section{References}

1. Engblom C, Pfirschke C and Pittet MJ: The role of myeloid cells in cancer therapies. Nat Rev Cancer 16: 447-462, 2016.

2. Banchereau J and Steinman RM: Dendritic cells and the control of immunity. Nature 392: 245-252, 1998.

3. James BR, Brincks EL, Kucaba TA, Boon L and Griffith TS Effective TRAIL-based immunotherapy requires both plasmacytoid and CD8 $\alpha$ dendritic cells. Cancer Immunol Immunother 63: 685-697, 2014.

4. Oppenheim JJ and Yang D: Alarmins: Chemotactic activators of immune responses. Curr Opin Immunol 17: 359-365, 2005.

5. Chiba S, Baghdadi M, Akiba H, Yoshiyama H, Kinoshita I, Dosaka-Akita H, Fujioka Y, Ohba Y, Gorman JV, Colgan JD, et al: Tumor-infiltrating DCs suppress nucleic acid-mediated innate immune responses through interactions between the receptor TIM-3 and the alarmin HMGB1. Nat Immunol 13: 832-842, 2012.

6. Scaffidi P, Misteli T and Bianchi ME: Release of chromatin protein HMGB1 by necrotic cells triggers inflammation. Nature 418: 191-195, 2002.

7. Tang D, Kang R, Zeh HJ III and Lotze MT: High-mobility group box 1 and cancer. Biochim Biophys Acta 1799: 131-140, 2010. 
8. Martinotti S, Patrone $\mathrm{M}$ and Ranzato E: Emerging roles for HMGB1 protein in immunity, inflammation, and cancer. Immunotargets Ther 4: 101-109, 2015.

9. Tang D, Kang R, Livesey KM, Cheh CW, Farkas A, Loughran P, Hoppe G, Bianchi ME, Tracey KJ, Zeh HJ III and Lotze MT: Endogenous HMGB1 regulates autophagy. J Cell Biol 190: 881-892, 2010

10. Campana L, Bosurgi L and Rovere-Querini P: HMGB1: A two-headed signal regulating tumor progression and immunity. Curr Opin Immunol 20: 518-523, 2008.

11. Levine B and Kroemer G: Autophagy in the pathogenesis of disease. Cell 132: 27-42, 2008

12. Mizushima N, Levine B, Cuervo AM and Klionsky DJ: Autophagy fights disease through cellular self-digestion. Nature 451: 1069-1075, 2008.

13. Kondo Y, Kanzawa T, Sawaya R and Kondo S: The role of autophagy in cancer development and response to therapy. Nat Rev Cancer 5: 726-734, 2005.

14. White E and DiPaola RS: The double-edged sword of autophagy modulation in cancer. Clin Cancer Res 15: 5308-5316, 2009.

15. Ogata M, Hino S, Saito A, Morikawa K, Kondo S, Kanemoto S, Murakami T, Taniguchi M, Tanii I, Yoshinaga K, et al: Autophagy is activated for cell survival after endoplasmic reticulum stress. Mol Cell Biol 26: 9220-9231, 2006.

16. Hoyer-Hansen $M$ and Jäättelä $M$ : Connecting endoplasmic reticulum stress to autophagy by unfolded protein response and calcium. Cell Death Differ 14: 1576-1582, 2007.

17. Li C, Capan E, Zhao Y, Zhao J, Stolz D, Watkins SC, Jin S and $\mathrm{Lu}$ B: Autophagy is induced in $\mathrm{CD} 4^{+} \mathrm{T}$ cells and important for the growth factor-withdrawal cell death. J Immunol 177 5163-5168, 2006

18. Kang R, Zeh HJ, Lotze MT and Tang D: The Beclin 1 network regulates autophagy and apoptosis. Cell Death Differ 18: 571-580, 2011.
19. Kang R, Livesey KM,Zeh HJ, Loze MT and Tang D: HMGB1: A novel Beclin 1-binding protein active in autophagy. Autophagy 6: 1209-1211, 2010.

20. Lutz MB, Kukutsch N, Ogilvie AL, Rössner S, Koch F, Romani N and Schuler G: An advanced culture method for generating large quantities of highly pure dendritic cells from mouse bone marrow. J Immunol Methods 223: 77-92, 1999.

21. Khong HT and Restifo NP: Natural selection of tumor variants in the generation of 'tumor escape' phenotypes. Nat Immunol 3: 999-1005, 2002.

22. Steinman RM and Banchereau J: Taking dendritic cells into medicine. Nature 449: 419-426, 2007.

23. Banchereau J and Palucka AK: Dendritic cells as therapeutic vaccines against cancer. Nat Rev Immunol 5: 296-306, 2005.

24. Kim R, Emi M and Tanabe K: Cancer immunoediting from immune surveillance to immune escape. Immunology 121: 1-14, 2007.

25. Liu L, Yang M, Kang R, Wang Z, Zhao Y, Yu Y, Xie M, Yin X, Livesey KM, Lotze MT, et al: HMGB1-induced autophagy promotes chemotherapy resistance in leukemia cells. Leukemia 25: 23-31, 2011.

26. Tang D and Lotze MT: Tumor immunity times out: TIM-3 and HMGB1. Nat Immunol 13: 808-810, 2012

27. Kusume A, Sasahira T, Luo Y, Isobe M, Nakagawa N, Tatsumoto N, Fujii K, Ohmori H and Kuniyasu H: Suppression of dendritic cells by HMGB1 is associated with lymph node metastasis of human colon cancer. Pathobiology 76: 155-162, 2009. 